\title{
WEIGHTED LEBESGUE AND LORENTZ NORM INEQUALITIES FOR THE HARDY OPERATOR
}

\author{
BY \\ ERIC SAWYER
}

\begin{abstract}
Characterizations are obtained for those pairs of weight functions $w, v$ for which the Hardy operator $T f(x)=\int_{0}^{x} f(s) d s$ is bounded from the Lorentz space $L^{r \cdot s}((0, \infty), v d x)$ to $L^{p \cdot q}((0, \infty), w d x), 0<p, q, r, s \leqslant \infty$. The modified Hardy operators $T_{\eta} f(x)=x^{-\eta} T f(x)$ for $\eta$ real are also treated.
\end{abstract}

1. Introduction. We characterize weighted Lebesgue and Lorentz norm inequalities for the Hardy operator $T f(x)=\int_{0}^{x} f(t) d t$ and the modified Hardy operators $T_{\eta} f(x)$ $=x^{-\eta} T f(x), \eta$ real, $x>0$ and $f$ nonnegative. For Lebesgue norms much is already known. For example if $1 \leqslant p \leqslant q \leqslant \infty$ we have the result of J. S. Bradley, K. Andersen, B. Muckenhoupt, M. Artola, G. Talenti and G. Tomaselli (see [1, 3, 5, 6, 7 and 8]) which states that

$$
\left(\int_{0}^{\infty} T f(x)^{q} w(x) d x\right)^{1 / q} \leqslant C\left(\int_{0}^{\infty} f(x)^{p} v(x) d x\right)^{1 / p} \quad \text { for all } f \geqslant 0
$$

if and only if the nonnegative weight functions $w, v$ satisfy

$$
\sup _{x>0}\left(\int_{x}^{\infty} w\right)^{1 / a}\left(\int_{0}^{x} v^{1-p^{\prime}}\right)^{1 / p^{\prime}}=A<\infty .
$$

Moreover, $A \leqslant C \leqslant p^{1 / q}\left(p^{\prime}\right)^{1 / p^{\prime}} A$ if $C$ is the least constant for which (1.1) holds. As usual we take $0 \cdot \infty=0,0^{0}=0$ and interpret $\left(\int_{a}^{b} v^{1-p^{\prime}}\right)^{1 / p^{\prime}}$ as $\left\|\chi_{(a, b)} v^{-1}\right\|_{L^{x}(v)}$ in the case $p^{\prime}=\infty$. The theorems below contain characterizations of (1.1) for all $p$ and $q$ satisfying $0<p, q \leqslant \infty$.

Our first theorem on weighted Lorentz norm inequalities (see below for definitions) for the Hardy operator $T$ makes use of a condition (see (1.4)) suggested by a recent work of H.-M. Chung, R. Hunt and D. Kurtz [2].

THEOREM 1. Suppose $0<p, q \leqslant \infty$ (where $q=\infty$ if $p=\infty$ ), $1<r<\infty$ and $1 \leqslant s \leqslant \infty$. If the weights $w, v \geqslant 0$ satisfy

$$
\|T f\|_{L^{p . q}(w)} \leqslant C\|f\|_{L^{r \cdot s}(v)} \text { for all } f \geqslant 0,
$$

Received by the editors July 26, 1982 and, in revised form, February 3, 1983.

1980 Mathematics Subject Classification. Primary 42B25.

${ }^{\prime}$ Research supported in part by the National Research Council of Canada. 
then the pair $w, v$ satisfies

$$
\begin{aligned}
\sup _{x>0}\left(\int_{x}^{\infty} w\right)^{1 / p}\left\|\chi_{(0, x)} v^{-1}\right\|_{L^{r^{\prime} v^{\prime}(v)}} & =A<\infty \\
\text { and } v & >0 \text { a.e. on }(0, x) \text { if } \int_{x}^{\infty} w>0 .
\end{aligned}
$$

Conversely, (1.4) implies (1.3) if and only if $q \geqslant \max \{r, s\}$.

This theorem leaves open the cases where $\min \{r, s\}<1$ or $q<\max \{r, s\}$. The next two theorems give partial results in each case. The first shows that if the basic index $r$ is less than one, then (1.3) holds if and only if the weight pair $w, v$ is trivial. The second theorem characterizes (1.3) for $q<\max \{r, s\}$ in the special case when $r=s$.

THEOREM 2. If $0<r<1$ and $0<p, q, s \leqslant \infty$, then (1.3) holds if and only if the weight pair $w, v$ is trivial in the sense that $v=\infty$ a.e. on any interval $(0, x)$ such that $\int_{x}^{\infty} w>0$.

Theorem 3. Suppose $0<p, q \leqslant \infty$ (where $q=\infty$ if $p=\infty$ ) and $1 \leqslant r<\infty$. Then

$$
\|T f\|_{L^{p .4(w)}} \leqslant C\|f\|_{L^{r}(v)} \text { for all } f \geqslant 0
$$

if and only if

$$
\sup _{x>0}\left(\int_{x}^{\infty} w\right)^{1 / p}\left(\int_{0}^{x} v^{1-r^{\prime}}\right)^{1 / r^{\prime}}=A<\infty
$$

in the case $q \geqslant r$ and if and only if

$$
\sup _{\ldots x_{k}<x_{k+1}}\left\{\sum_{k}\left[\left(\int_{x_{k}}^{x_{k+1}} \tilde{w}\right)^{1 / q}\left(\int_{x_{k-1}}^{x_{k}} v^{1-r^{\prime}}\right)^{1 / r^{\prime}}\right]^{\rho}\right\}^{1 / \rho}=B<\infty,
$$

where $1 / \rho=1 / q-1 / r$ in the case $0<q<r$. The sup in (1.7) is taken over all positive increasing sequences $\left\{x_{k}\right\}$ and

$$
\tilde{w}(x)=-\frac{d}{d x}\left(\int_{x}^{\infty} w\right)^{q / p}=\frac{q}{p}\left(\int_{x}^{\infty} w\right)^{(q / p)-1} w(x) .
$$

We now turn to the modified Hardy operators $T_{\eta} f(x)=x^{-\eta} T f(x)$. Andersen and Muckenhoupt have characterized weighted weak type inequalities for these operators [5]. For $\eta \leqslant 0, T_{\eta} f$ satisfies the following monotonicity condition $-T_{\eta} f$ is nondecreasing for nonnegative $f$-and this allows us to replace weighted Lorentz norms of $T_{\eta} f$ by weighted Lebesgue norms of $T f$ and Theorems 1-3 can now be used. Indeed, for $f \geqslant 0,(2.6)$ below shows that the $L^{p . q}(w)$ norm of $T_{\eta} f$ coincides with the $L^{q}\left(\tilde{w}_{\eta}\right)$ norm of $T f$ where $\tilde{w}_{\eta}(x)=x^{-\eta q} \tilde{w}(x)$ and $\tilde{w}$ is as in Theorem 3 .

For $\eta>0, T_{\eta}$ does not satisfy the above monotonicity condition and, consequently, weighted Lorentz norms of $T_{\eta} f$ are harder to deal with (Lebesgue norms of $T_{\eta} f$ are of course equal to weighted Lebesgue norms of $T f$ ). There is, however, one case in which a weighted Lorentz norm inequality for $T_{\eta} f, \eta>0$, can be reduced by duality to an inequality for an operator satisfying the monotonicity condition. The case $q=\infty$ of the following theorem is contained in [5]. 
TheOREM 4. Suppose $\eta>0$ and $1 \leqslant r \leqslant \min \{p, q\}$. Then

$$
\left\|T_{\eta} f\right\|_{L^{p . q(w)}} \leqslant C\|f\|_{L^{r}(v)} \text { for all } f \geqslant 0
$$

if and only if

$$
\left\|s^{-\eta} \chi_{(x, \infty)}(s)\right\|_{L^{p .4}(w)}\left(\int_{0}^{x} v^{1-r^{\prime}}\right)^{1 / r^{\prime}} \leqslant C \quad \text { for all } x>0,
$$

where the second factor on the left side of (1.9) is interpreted as $\left\|\chi_{(0, x)} v^{-1}\right\|_{L^{\infty}(v)}$ if $r=1$.

Note that in the case $q=\infty,(1.9)$ becomes

$$
\sup _{0<x \leqslant s<\infty} s^{-\eta}\left(\int_{x}^{s} w\right)^{1 / p}\left(\int_{0}^{x} v^{1-r^{\prime}}\right)^{1 / r^{\prime}} \leqslant C,
$$

which is easily seen to coincide with the condition of Andersen and Muckenhoupt in [5, Theorem 2]. It may be useful to point out that for $q<\infty$,

$$
\left\|s^{-\eta} \chi_{(x, \infty)}\right\|_{L^{p .4(w)}}^{q}=\eta q \int_{x}^{\infty}\left[s^{-\eta}\left(\int_{x}^{s} w\right)^{1 / p}\right]^{q} \frac{d s}{s} .
$$

We now give some definitions. If $f$ denotes a measurable function defined on a measure space $(M, \mu)$, the distribution function $f_{*}$ and the nonincreasing rearrangement $f^{*}$ of $f$ with respect to $\mu$ are given by (see e.g. [4, Chapter V])

$$
f_{*}(s)=|\{|f|>s\}|_{\mu}=\int_{\{|f|>s\}} d \mu, \quad f^{*}(t)=\inf \left\{s ; f_{*}(s) \leqslant t\right\} .
$$

For $0<p<\infty, 0<q \leqslant \infty$, the Lorentz space $L^{p, q}(\mu)$ consists of all functions $f$ satisfying $\|f\|_{L^{p . q}(\mu)}<\infty$, where

$$
\|f\|_{L^{p . q}(\mu)}= \begin{cases}{\left[\int_{0}^{\infty} \frac{q}{p} t^{q / p-1} f^{*}(t)^{q} d t\right]^{1 / q}} & \text { for } 0<q<\infty \\ \sup _{t>0} t^{1 / p} f^{*}(t) & \text { for } q=\infty\end{cases}
$$

Note that

$$
\|f\|_{L^{p, p}(\mu)}=\|f\|_{L^{p}(\mu)}=\left(\int_{M}|f|^{p} d \mu\right)^{1 / p} .
$$

We shall need the following basic relationship between $L^{p, q}$ and $L^{p^{\prime}, q^{\prime}}$, where $1 / p+1 / p^{\prime}=1=1 / q+1 / q^{\prime}, 1<p<\infty$ and $1 \leqslant q \leqslant \infty$. (See e.g. [2, inequality (2.3)].)

$$
C^{-1}\|f\|_{L^{p, q}(\mu)} \leqslant \sup _{\|g\|_{L^{p . q^{\prime}}(\mu)} \leqslant 1}\left|\int f g d \mu\right| \leqslant C\|f\|_{L^{p, q}(\mu)} .
$$




\section{Proofs of theorems.}

Proof of Theorem 1. Applying the change of variable $t=f_{*}(s)$ to the right side of (1.10) and integrating by parts we obtain [2, (2.4)]

$$
\|f\|_{L^{p \cdot q}(\mu)}= \begin{cases}{\left[\int_{0}^{\infty} q s^{q-1} f_{*}(s)^{q / p} d s\right]^{1 / q}} & \text { for } 0<q<\infty \\ \sup _{s>0} s f_{*}(s)^{1 / p} & \text { for } q=\infty\end{cases}
$$

(or simply evaluate the two iterated integrals of $q s^{q-1}(q / p) t^{q / p-1}$ over the set $\left.\left\{(t, s) ; 0<s<f^{*}(t), 0<t\right\}\right)$. We now prove (1.3) $\Rightarrow(1.4)$. Let $f$ be nonnegative on $(0, \infty)$. Then $T\left(\chi_{(0, x)} f\right) \geqslant \int_{0}^{x} f$ on $[x, \infty)$ and so $T\left(\chi_{(0, x)} f\right)_{*}(\xi) \geqslant \int_{x}^{\infty} w$ for $0 \leqslant \xi<$ $\int_{0}^{x} f$. Inequality (1.3), together with (2.1), yields

$$
\begin{aligned}
\|f\|_{L^{r \cdot v}(v)} & \geqslant C^{-1}\|T f\|_{L^{p \cdot u}\left(u^{*}\right)} \\
& \geqslant C^{-1}\left[\int_{0}^{\lambda}\left(\int_{x}^{x} w\right)^{q / p} q t^{q-1} d t\right]^{1 / q} \quad\left(\lambda=\int_{0}^{x} f\right) \\
& =C^{-1}\left(\int_{x}^{\infty} w\right)^{1 / p}\left(\int_{0}^{x} f\right) \geqslant C^{-1}\left(\int_{x}^{\infty} w\right)^{1 / p}\left(\int_{0}^{x} f v^{-1} v\right)
\end{aligned}
$$

and (1.4) now follows easily upon using (1.11) for $0<p, q<\infty$. The cases $p=\infty$ or $q=\infty$ are established by simple modifications of this argument.

Conversely, fix $f \geqslant 0$ in $L^{r . s}(v)$ and suppose $q \geqslant \max \{r, s\}$. If $w \neq 0$, then $\int_{x}^{\infty} w>0$ for some $x>0$ and from (1.4) and (1.11) we have

$$
\int_{0}^{x} f=\int_{0}^{x} f v^{-1} v \leqslant\|f\|_{L^{r \cdot s}(v)}\left\|\chi_{(0, x)} v^{-1}\right\|_{L^{r^{\prime \prime}(v)}}<\infty
$$

Thus we can choose $x_{k}$ such that $T f\left(x_{k}\right)=\int_{0}^{x_{k}} f=2^{k}$ for all $k$ in $Z$ satisfying $2^{k}<\int_{0}^{\infty} f$. We suppose $0<q<\infty$, the case $q=\infty$ being an easy modification of the following argument. From (2.1) we have

$$
\begin{aligned}
& \|T f\|_{L^{p \cdot 4\left(w^{\prime}\right)}}^{q}=q \int_{0}^{\infty} s^{q-1}(T f)_{*}(s)^{q / p} d s \leqslant C \sum_{k} 2^{k q}\left(\int_{\left\{T f>2^{k}\right\}} w\right)^{q / p} \\
& \leqslant C \sum_{k}\left(\int_{x_{k-1}}^{x_{k}} f v^{-1} v\right)^{q}\left(\int_{x_{k}}^{x} w\right)^{q / p} \\
& \leqslant C \sum_{k}\left\|f_{k}\right\|_{L^{\prime, s}(v)}^{q}\left\|\chi_{\left(0, x_{k}\right)} v^{-1}\right\|_{L^{r^{\prime \prime s}(v)}}^{q}\left(\int_{x_{k}}^{\infty} w\right)^{q / p}
\end{aligned}
$$

by (1.11), where

$$
f_{k}=\chi_{\left(x_{\left.k-1, x_{k}\right)}\right.} f \leqslant C A \sum_{k}\left\|f_{k}\right\|_{L^{r \cdot s}(v)}^{q} \leqslant C A\|f\|_{L^{r \cdot s}(v)}^{q}
$$

by the following lemma, which is a slight extension of Lemma 2.5 in [2]. 
Lemma 1 [2]. Let $(M, \mu)$ be a measure space. Suppose $q \geqslant \max \{r, s\}$ and $\left\{E_{k}\right\}$ is a sequence of disjoint measurable subsets of $M$. Then

$$
\sum_{k}\left\|\chi_{E_{k}} f\right\|_{I^{r \cdot 1}(\mu)}^{q} \leqslant\|f\|_{L^{r \cdot 1}(\mu)}^{q} .
$$

ProOF.

$$
\begin{aligned}
\sum_{k}\left\|\chi_{E_{k}} f\right\|_{L^{r \cdot s}(\mu)}^{q r \cdot} & =\sum_{k}\left(\int_{0}^{\infty}\left(\chi_{E_{k}} f\right)_{*}(t)^{s / r} s t^{s-1} d t\right)^{q / s} \\
& \leqslant\left(\int_{0}^{\infty}\left(\sum_{k}\left(\chi_{E_{k}} f\right)_{*}(t)^{q / r}\right)^{s / q} s t^{s-1} d t\right)^{q / s} \\
& \leqslant\left(\int_{0}^{\infty} f_{*}(t)^{s / r} s t^{s-1} d t\right)^{q / s}=\|f\|_{L^{r . s}(\mu)}^{q} \quad \text { since } q \geqslant r .
\end{aligned}
$$

It remains to show that if $q<\max \{r, s\}$, then (1.4) does not imply (1.3). We consider two cases: $q<s$ and $s \leqslant q<r$. If $q<s$, set $v \equiv 1$ on $(0, \infty)$ and let $w$ be such that the product on the left side of (1.4) is identically 1, i.e. $w(x)=$ $\left(p / r^{\prime}\right) x^{-p / r^{\prime}-1}$. Let

$$
f_{\alpha, \beta}(x)=x^{-\alpha}(1+|\log x|)^{-\beta},
$$

where $\alpha=1 / r$ and $q<1 / \beta<s$. Now if $x_{t}=t^{-1 / \alpha}(1+|\log t|)^{-\beta / \alpha}$, then $f_{\alpha \beta}\left(x_{t}\right) \approx t$, so using (2.1),

$$
\begin{aligned}
\left\|f_{\alpha \beta}\right\|_{L^{r s}(v)}^{s} & =s \int_{0}^{\infty}\left|\left\{f_{\alpha, \beta}>t\right\}\right|^{s / r} t^{s-1} d t \\
& \approx \int_{0}^{\infty} x_{t}^{s / r} t^{s-1} d t=\int_{0}^{\infty} \frac{d t}{t(1+|\log t|)^{\beta s}}<\infty .
\end{aligned}
$$

On the other hand,

$$
T f_{\alpha \beta}(x) \approx x^{1 / r^{\prime}}(1+|\log x|)^{-\beta}
$$

and for $y_{t}=t^{r^{\prime}}(1+|\log t|)^{\beta r^{\prime}}$, we have $T f_{\alpha \beta}\left(y_{t}\right) \approx t$. Thus

$$
\begin{aligned}
\left\|T f_{\alpha \beta}\right\|_{L^{p \cdot q(w)}}^{q} & =q \int_{0}^{\infty}\left|\left\{T f_{\alpha \beta}>t\right\}\right|_{w^{\prime}}^{q / p} t^{q-1} d t \\
& \approx \int_{0}^{\infty}\left[\int_{y_{t}}^{\infty} \frac{p}{r^{\prime}} x^{-p / r^{\prime}-1} d x\right]^{q / p} t^{p-1} d t \\
& =\int_{0}^{\infty} \frac{d t}{t(1+|\log t|)^{\beta q}}=\infty
\end{aligned}
$$

so the weight pair $(w, v)$ satisfies (1.4) but not (1.3).

Finally, if $s \leqslant q<r$, set $v(x)=f_{\alpha, \beta}(x)$, where $\alpha=1$ and $1<\beta<r / q$, and let $w(x)=f_{\gamma, \delta}(x)$, where $\gamma=p+1$ and $\delta=\beta p / r$. Then

$$
\left(\int_{x}^{\infty} w\right)^{1 / p} \approx x^{-1}(1+|\log x|)^{-\beta / r}
$$


and for $s \leqslant r$ we have

$$
\begin{aligned}
\left\|\chi_{(0, x)} v^{-1}\right\|_{L^{r^{\prime} x^{\prime}(v)}} & \leqslant\left\|\chi_{(0, x)} v^{-1}\right\|_{L^{r^{\prime}(v)}}=\left(\int_{0}^{x} v^{-r^{\prime} v}\right)^{1 / r^{\prime}} \\
& \leqslant C x(1+|\log x|)^{\beta / r}
\end{aligned}
$$

so condition (1.4) holds for the weights $w, v$. With $f \equiv 1$ on $(0, \infty)$ we have

$$
\|f\|_{L^{r \cdot s}(v)}^{s}=\left(\int_{0}^{\infty} v\right)^{s / r}<\infty
$$

since $\beta>1$, while

$$
\begin{aligned}
\|T f\|_{L^{p . q\left(x^{\prime}\right)}}^{q} & =q \int_{0}^{\infty}|\{T f>t\}|_{k^{\prime}}^{q / p} t^{q-1} d t \\
& \approx \int_{0}^{\infty} \frac{d t}{t(1+|\log t|)^{\beta p / r}}=\infty \quad \text { since } \frac{\beta q}{r}<1,
\end{aligned}
$$

so (1.4), but not (1.3), holds for the weight pair $(w, v)$. This completes the proof of Theorem 1.

Proof of Theorem 2. Clearly (1.3) holds if the weight pair $(w, v)$ is trivial in the sense indicated in Theorem 2. Conversely suppose (1.3) holds for some $r<1$. Let $F(x)=\min \left\{1, v(x)^{-1 / r}\right\}$ and set $f_{a, b}=\chi_{(a, b)} F$ for $0<a<b<\infty$. Suppose for the moment that $r \leqslant s$ and $p<\infty$. With $f=f_{a, b}$ in (1.3) we obtain, as in (2.2), that

$$
\begin{aligned}
\left(\int_{a}^{b} F\right)\left(\int_{h}^{\infty} w\right)^{1 / p} & \leqslant C\left\|f_{a, b}\right\|_{L^{r \cdot \cdot(v)}} \\
& \leqslant C\left\|f_{a, b}\right\|_{L^{r}(v)}=C\left(\int_{a}^{h} F^{r} v\right)^{1 / r} \text { since } r \leqslant s \\
& \leqslant C(b-a)^{1 / r} \text { since } F(x)^{r} v(x) \leqslant 1 \text { for all } x .
\end{aligned}
$$

Now divide both sides of $(2.4)$ by $(b-a)$ and let $(a, b)$ shrink to a Lebesgue point $x$ of $F$ to obtain $F(x)\left(\int_{x}^{\infty} w\right)^{1 / p} \leqslant 0$, which yields $v(x)=\infty$ if $\int_{x}^{\infty} w>0$. If $0<s<r$ we can modify the above argument as follows. Let $d=\inf \left\{x ; \int_{x}^{\infty} w=0\right\}$. If $v$ is not infinite a.e. on $(0, d)$ then there is a set $E \subset(0, d)$ of positive Lebesgue measure satisfying $\int_{E} v<\infty$. Suppose, in order to derive a contradiction, that such a set $E$ exists. Let $F(x)$ be as above but set $f_{a, b}=\chi_{E \cap(a, b)} F$ for $0<a<b<d$. Now choose $\rho$ such that $d<\rho<1$ and in (2.4) replace $\left\|f_{a, b}\right\|_{L^{r \cdot s}(v)} \leqslant C\left\|f_{a, b}\right\|_{L^{r}(v)}$ (which may fail for $s<r$ ) with $\left\|f_{a, b}\right\|_{L^{r . s}(v)} \leqslant C^{\prime}\left\|f_{a, b}\right\|_{L^{\rho}(v)}$, where $C^{\prime}$ depends on $\int_{E} v$ and $\rho$ as well as on $r$ and $s$. Arguing as before we obtain that $\chi_{E}(x) F(x)\left(\int_{x}^{\infty} w\right)^{1 / p} \leqslant 0$ whenever $x$ is a Lebesgue point of $\chi_{E} F$. Since $\int_{x}^{\infty} w>0$ for $x<d$ we conclude that $v=\infty$ a.e. on $E$. Thus $\int_{E} v=\infty$, the desired contradiction. The case $p=\infty$ is an easy adaptation of these arguments and this completes the proof of Theorem 2.

Proof of TheOReM 3. In the case $q \geqslant r>1$, the equivalence of (1.5) and (1.6) is a special case of Theorem 1. If $q \geqslant r=1$ the equivalence of (1.5) and (1.6) can be established by the argument of Theorem 1 (see (2.2) and (2.3)) since the analogue of 
(1.11) holds in this case, i.e.

$$
\|f\|_{L^{r}(v)}=\sup _{\|g\|_{L^{\prime}(v)} \leqslant 1}\left|\int f g v\right| \text { for } 1 \leqslant r \leqslant \infty .
$$

To handle the case $0<q<r$, we first observe that if $h$ is nonnegative and nondecreasing on $(0, \infty)$ then

$$
\|h\|_{L^{p . q}(w)}^{q}=\frac{q}{p} \int_{0}^{\infty} h(x)^{q} g(x)^{q / p-1} w(x) d x,
$$

where $g(x)=\int_{x}^{\infty} w$. This equality is established by evaluating the two iterated integrals of $q s^{q-1}(q / p) g(x)^{q / p-1} w(x)$ over the set $\{(x, s) ; 0<s<h(x), 0<x\}$. Performing the $s$ integration first yields the right side of (2.6), and performing the $x$ integration first yields the right side of (2.1) since for fixed $s$, if

$$
x(s)=\sup \{x ; h(x) \leqslant s\}
$$

then

$$
\int_{x(s)}^{\infty} \frac{q}{p} g(x)^{q / p-1} w(x) d x=g(x(s))^{q / p}=\left(\int_{x(s)}^{\infty} w\right)^{q / p}=h_{*}(s)^{q / p} .
$$

Now suppose (1.5) holds and $0<q<r$. First assume $r>1$. Fix a positive increasing sequence $\cdots x_{k}<x_{k+1} \cdots$ and, given a sequence of positive numbers $a_{k}$, set $f=\sum_{k} a_{k} \chi_{k} \sigma$, where $\chi_{k}=\chi_{\left(x_{k-1}, x_{k}\right)}$ and $\sigma=v^{1-r^{\prime}}$. Then with $\tilde{w}(x)=$ $(q / p) g(x)^{q / p-1} w(x)$ we have

$$
\begin{aligned}
\left(\sum_{k} a_{k}^{r} \int \chi_{k} \sigma\right)^{q / r} & =\left\|\sum_{k} a_{k} x_{k} \sigma\right\|_{L^{\prime}(v)}^{q} \geqslant C\|T f\|_{L^{p . q}(w)}^{q} \\
& =C \frac{q}{p} \int_{0}^{\infty} T f(x)^{q} g(x)^{q / p-1} w(x) d x \text { by }(2.6) \\
& \geqslant C \sum_{k} T f\left(x_{k}\right)^{q} \int_{x_{k}}^{x_{k+1}} \frac{q}{p} g(x)^{q / p-1} w(x) d x \\
& \geqslant C \sum_{k}\left(\int_{x_{k-1}}^{x_{k}} f\right)^{q}\left(\int_{x_{k}}^{x_{k+1}} \tilde{w}\right) \\
& =C \sum_{k}\left[a_{k}^{q}\left(\int x_{k} \sigma\right)^{q / r}\right]\left[\left(\int_{x_{k}}^{x_{k+1}} \tilde{w}\right)\left(\int_{x_{k-1}}^{x_{k}} v^{1-r^{\prime}}\right)^{q / r^{\prime}}\right]
\end{aligned}
$$

for all sequences $\left\{a_{k}\right\}$ of nonnegative numbers. Since the dual of the sequence space $l^{r / q}$ is $l^{(r / q)^{\prime}},(2.7)$ shows that the sequence, whose terms consist of the second factors $[\cdots]$ in the final sum above, is in $l^{(r / q)^{\prime}}$. Since $q(r / q)^{\prime}=\rho$, this proves (1.7). Note that (1.7) persists even if $\int \chi_{k} \sigma=\infty$ for some $k$ since then (1.5) easily implies $\int_{x_{k}}^{\infty} w=0$. Finally for $r=1$ we modify this argument as follows. Let $f=\Sigma_{k} a_{k} f_{k}$, where $f_{k}$ is supported in $\left[x_{k-1}, x_{k}\right]$. If now $f_{k}$ is allowed to vary within the unit ball of $L^{1}(v),(2.7)$ and (2.5) yield (1.7) as above.

Now suppose (1.7) holds and $0<q<r$. Fix $f \geqslant 0$ in $L^{r}(v)$. As in the proof of Theorem 1 we can choose $x_{k}$ such that $T f\left(x_{k}\right)=2^{k}$ for all integers $k$ satisfying 
$2^{k}<\int_{0}^{\infty} f$. Then (2.6) yields

$$
\begin{aligned}
\|T f\|_{L^{p . q}(w)}^{q} & =\frac{q}{p} \int_{0}^{\infty} T f(x)^{q} g(x)^{q / p-1} w(x) d x=\sum_{k} \int_{x_{k}}^{x_{k+1}} \\
& \leqslant 2^{q} \sum_{k} 2^{k q}\left(\int_{x_{k}}^{x_{k+1}} \tilde{w}\right) \leqslant 4^{q} \sum_{k}\left(\int_{x_{k-1}}^{x_{k}} f\right)^{q}\left(\int_{x_{k}}^{x_{k+1}} \tilde{w}\right) \\
& \leqslant 4^{q} \sum_{k}\left(\int_{x_{k-1}}^{x_{k}} f^{r} v\right)^{q / r}\left[\left(\int_{x_{k-1}}^{x_{k}} v^{1-r^{\prime}}\right)^{1 / r^{\prime}}\left(\int_{x_{k}}^{x_{k+1}} \tilde{w}\right)^{1 / q}\right]^{q} \\
& \leqslant 4^{q} B^{q}\left(\sum_{k} \int_{x_{k-1}}^{x_{k}} f^{r} v\right)^{q / r}=\left(4 B\|f\|_{L^{r}(v)}\right)^{q}
\end{aligned}
$$

by an application of Holder's inequality with exponents $r / q$ and $(r / q)^{\prime}$. Thus (1.5) holds and this completes the proof of Theorem 3 .

Proof of Theorem 4. Let $f$ be nonnegative on $(0, \infty)$ and fix $x>0$. Since $T_{\eta} f(s) \geqslant s^{-\eta} \int_{0}^{x} f$ for $s \geqslant x$, we have, from (1.8),

$$
\left(\int_{0}^{x} f v^{-1} v\right)\left\|s^{-\eta} \chi_{(x, \infty)}\right\|_{L^{p . q}(w)} \leqslant\left\|T_{\eta} f\right\|_{L^{p . q}(w)} \leqslant C\|f\|_{L^{r}(v)} .
$$

By duality we obtain

$$
\left\|\chi_{(0, x)} v^{-1}\right\|_{L^{r^{\prime}(v)}}\left\|s^{-\eta} \chi_{(x, \infty)}\right\|_{L^{p . q(w)}} \leqslant C
$$

which is (1.9).

Conversely, we begin by noting that (1.8) is equivalent to the dual inequality

$$
\left\|v^{-1} T_{\eta}^{*}(g w)\right\|_{L^{r^{\prime}(v)}} \leqslant C\|g\|_{L^{p^{\prime}, q^{\prime}(w)}} \text { for all } g \geqslant 0
$$

where $T_{\eta}^{*} f(x)=\int_{x}^{\infty} s^{-\eta} f(s) d s$. Suppose $g \geqslant 0$ and $\|g\|_{L^{p^{\prime} \cdot q^{\prime}(w)}}<\infty$. Consider first the case $r>1$. Provided $v \not \equiv \infty$ on $(0, \infty)$ we can, as in the proof of Theorem 1 , choose $x_{k}$ such that $\int_{x_{k}}^{\infty} s^{-\eta} g(s) w(s) d s=2^{k}$ for all integers $k$ satisfying $2^{k}<$ $\int_{0}^{\infty} s^{-\eta} g w d s$.

Then with $\chi_{k}=\chi_{\left(x_{k}, x_{k-1}\right)}$ we have

$$
\begin{aligned}
\left\|v^{-1} T_{\eta}^{*}(g w)\right\|_{L^{r^{\prime}(v)}}^{r^{\prime}} & \leqslant C \sum_{k}\left(\int_{x_{k}}^{x_{k-1}} s^{-\eta} g w d s\right)^{r^{\prime}}\left(\int_{0}^{x_{k}} v^{1-r^{\prime}}\right) \\
& \leqslant C \sum_{k}\left\|\chi_{k} g\right\|_{L^{p^{\prime}, q^{\prime}(w)}}^{r^{\prime}}\left[\left\|s^{-\eta} \chi_{\left(x_{k}, \infty\right)}(s)\right\|_{L^{p \cdot q(w)}}^{r^{\prime}} \int_{0}^{x_{k}} v^{1-r^{\prime}}\right] \\
& \leqslant C^{\prime} \sum_{k}\left\|\chi_{k} g\right\|_{L^{p^{\prime} \cdot q^{\prime}(w)}}^{r^{\prime}} \text { by }(1.9) \\
& \leqslant C^{\prime}\|g\|_{L^{p^{\prime} \cdot q^{\prime}(w)}}^{r^{\prime}}
\end{aligned}
$$


by Lemma 1 since $r^{\prime} \geqslant \max \left\{p^{\prime}, q^{\prime}\right\}$. Finally for $r=1$ we have

$$
\begin{aligned}
\left\|v^{-1} T_{\eta}^{*}(g w)\right\|_{L^{x}(v)} & \leqslant \sup _{x>0}\left\|\chi_{(0, x)} v^{-1}\right\|_{L^{x}(v)} T_{\eta}^{*}(g w)(x) \\
& =\sup _{x>0}\left\|\chi_{(0, x)} v^{-1}\right\|_{L^{x}(v)} \int_{x}^{\infty} s^{-\eta} g(s) w(s) d s \\
& \leqslant \sup _{x>0}\left\|\chi_{(0, x)} v^{-1}\right\|_{L^{\infty}(v)}\left\|s^{-\eta} \chi_{(x, x)}(s)\right\|_{L^{p .4}(w)}\|g\|_{\left.L^{p^{\prime} \cdot u^{\prime}\left(x^{\prime}\right)}\right)} \\
& \leqslant C\|g\|_{L^{p^{\prime}, u^{\prime}\left(x^{\prime}\right)}} \text { by }(1.9),
\end{aligned}
$$

and this completes the proof of Theorem 4.

Remarks. (I) Theorem 3 has a simple analogue in the case $r=\infty$. If $0<p, q \leqslant \infty$ (where $q=\infty$ if $p=\infty$ ) then

$$
\|T f\|_{L^{n \cdot 4\left(x^{*}\right)}} \leqslant C\|f\|_{L^{x}(\varepsilon)} \text { for all } f \geqslant 0
$$

if and only if $\int_{x}^{\infty} w=0$ whenever $\mid\{t$ in $[0, x] ; v(t)=0\} \mid$ is positive and $\|h\|_{I^{p \cdot q}\left(n^{*}\right)}$ $\leqslant C$, where $h(x)=x$ for $x>0$.

(II) In the case $q=r$ of Theorem 3, one has $A \leqslant C \leqslant q^{1 / q}\left(q^{\prime}\right)^{1 / q^{\prime}} A$ provided $C$ is the least constant for which (1.5) holds. These inequalities are sharp. That $A \leqslant C$ is obvious and to obtain the other inequality replace $h$ by $T f$ in (2.6) and apply Theorem 1 of [3] (i.e. the equivalence of (1.1) and (1.2) for $p=q$ ) to obtain (1.5) with

$$
\begin{aligned}
C & \leqslant q^{1 / q}\left(q^{\prime}\right)^{1 / q^{\prime}} \sup _{x>0}\left(\int_{x}^{\infty} \frac{q}{p} g(t)^{q / p-1} w(t) d t\right)^{1 / q}\left(\int_{0}^{x} v^{1-q^{\prime}}\right)^{1 / q^{\prime}} \\
& =q^{1 / q}\left(q^{\prime}\right)^{1 / q^{\prime}} A .
\end{aligned}
$$

To show that this latter inequality is best possible let $w(x)=x^{-p / q^{\prime}-1}$ and $v(x)=1$ so that $A$ in (1.6) equals $\left(p / q^{\prime}\right)^{1 / p}$. For $\delta>-1 / q$ define $f_{\delta}(x)=\chi_{(0, t)}(x) x^{\delta}$, where $t$ is chosen so that $\left\|f_{\delta}\right\|_{L^{4}}=1$, i.e. $t=(1+q \delta)^{1 / 1+q \delta}$. A computation using (2.6) shows that

$$
\lim _{\delta \rightarrow-1 / q}\left\|T f_{\delta}\right\|_{L^{p \cdot q(w)}}=\left(q^{\prime} / p\right)^{1 / p} q^{1 / q}\left(q^{\prime}\right)^{1 / q^{\prime}}=A q^{1 / q}\left(q^{\prime}\right)^{1 / q^{\prime}}
$$

\section{REFERENCES}

1. J. S. Bradley, Hardy inequalities with mixed norms, Canad. Math. Bull. 21 (1978), 405-408.

2. H.-M. Chung, R. A. Hunt and D. S. Kurtz, The Hardy-Littlewood maximal function on L $(p, q)$ spaces with weights, preprint.

3. B. Muckenhoupt, Hardy's inequality with weights, Studia Math. 34 (1972), 31-38.

4. E. M. Stein and G. Weiss, Fourier analysis on Euclidean spaces, Princeton Univ. Press, Princeton, N.J., 1971.

5. K. Andersen and B. Muckenhoupt, Weighted weak type Hardy inequalities with applications to Hilhert transforms and maximal functions, Studia Math. 72 (1982), 9-26.

6. M. Artola, untitled and unpublished manuscript.

7. G. Talenti, Osservazioni sopra una classe di disuguaglianze, Rend. Sem. Mat. Fis. Milano 39 (1969), 171-185.

8. G. Tomasselli, A class of inequalities, Boll. Un. Mat. Ital. 21 (1969), 622-631.

Department of Mathematical Sciences, McMaster University, Hamilton, Ontario, Canada L8S 4K 1 